\title{
Mineralogía y cambios composicionales en fragmentos óseos atribuidos a un dinosaurio ornitópodo del yacimiento barremiense de Buenache de la Sierra (Formación Calizas de La Huérguina, Cuenca, España).
}

\author{
Mineralogy and compositional changes in bone fragments attributed \\ to an ornithopod dinosaur from the Barremian locality of Buenache de \\ la Sierra (La Huerguina limestones Formation, Cuenca, Spain).
}

\author{
L. Merino ${ }^{1}$, A.D. Buscalioni²
}

\begin{abstract}
RESUMEN
Las diferentes técnicas utilizadas (difracción de rayos $\mathrm{X}$, microscopio petrográfico, microscopio electrónico de barrido y microsonda electrónica) en el estudio de los restos óseos atribuidos a un ornitópodo del yacimiento Buenache de la Sierra (Baremiense superior, Formación La Huérguina) han permitido caracterizar los procesos diagenéticos. Se han analizado huesos de arcosaurios vivos (caimán y ave), para comparar la mineralogía y la geoquímica del material óseo. Los procesos diagenéticos han modificado la composición química original de los fragmentos de huesos estudiados, así como su cristalinidad, que aumenta en el caso de las muestras fósiles. En las costillas de los taxones actuales estudiados la fase mineral predominante es el hidroxiapatito y en las fósiles el fluorapatito. Encontramos dos tipos de organización mineralógica: un grupo presenta la estructura esponjosa totalmente colapsada, formando una brecha de esquirlas, mientras que en el segundo grupo la conservación histológica es evidente, aunque hay sustituciones parciales por carbonato cálcico. En el material óseo fósil el mineral autigénico más abundante es la calcita, que rellena los poros y fracturas. La presencia de restos de pirita en las cavidades del hueso indica que éstos se conservaron en un ambiente reductor en una etapa temprana del enterramiento. El estudio de los cambios bioestratinómicos y fosildiagenéticos nos permite concluir que se trata de una asociación resedimentada, con restos que han sufrido distintas historias tafonómicas.
\end{abstract}

Palabras clave: cristalinidad, cementación, dinosaurio, mineral autigénico, remoción tafonómica

\begin{abstract}
X-ray diffraction, optical polarizing microscopy, scanning electron microscopy and electron microprobeto, have been used to analyse the diagenetic processes of isolated dinosaur bones and ribs attributed to an ornithopod dinosaur from Buenache de la Sierra (Upper Barremian, La Huérguina Formation). We also examine the geochemical and mineralogical bone mineralization of extant archosaurs (caiman and bird) for comparison. Diagenetic processes have modified the original chemical composition of the studied fragments of bones, as well as its crystallinity, which increases in the fossil samples. In recent bones, hydroxyapatite results the main mineral constituent, while in fossils is fluorapatite. We found two types of mineralogical arrangements: one group exhibiting the spongy structure completely collapsed forming breccias of bone fragments, and a second group with well preserved spongy structure but substituted by calcium carbonate. Calcite is the most abundant authigenic mineral infilling pores and fissures of the fossilized remains. The presence of pyrite in bone cavities suggests an early burial stage under euxinic conditions of a reductor environment. The
\end{abstract}

\footnotetext{
${ }^{1}$ Departamento de Paleobiología. Museo Nacional de Ciencias Naturales. José Gutiérrez Abascal, 2. 28006 Madrid, España. Email: mcnlm600@mncn.csic.es

2 Unidad de Paleontología, Departamento Biología, Facultad Ciencias Universidad Autónoma de Madrid, 28049 Cantoblanco, Madrid, España. angela.delgado@uam.es
} 
biostratinomic and fossildiagenetic alterations allow us to conclude that the remains have two distinct taphonomic histories, and that the association undergone taphonomical resedimentation.

Keywords: authigenic mineral, crystallinity, cementation, dinosaur, taphonomic removal

\section{Introducción}

La composición elemental de los huesos y dientes de los organismos cambia a lo largo de su vida. Estos cambios se producen principalmente por distintos factores como son las condiciones ambientales, dieta, edad y enfermedades (Driessens \& Verbeeck, 1990). El material óseo es un hidroxiapatito $\left[\mathrm{Ca}_{5}\left(\mathrm{PO}_{4}\right)_{3}(\mathrm{OH})\right]$ en el que ocurren numerosas sustituciones iónicas dentro de su estructura cristalina, tanto en vida como después de la muerte durante los procesos de fosilización (Goodwin et al., 2007). Estos cambios químicos llegan incluso a afectar de una manera significativa a los esmaltes que, en principio, por tener una estructura compacta deberían conservar mejor la composición original. Por ejemplo, una ingesta prolongada de agua con alto contenido en plomo puede verse reflejada en un aumento de su composición en los huesos e incluso en los esmaltes de los dientes (Moore et al., 1978; Aguinaga et al., 2000).

Una vez enterrado el hueso fresco el colágeno y otras proteínas (40\% de su volumen) se degradan y la porosidad resultante se puede rellenar con minerales autigénicos durante el proceso de fosilización (Henderson et al., 1983; Dauphin \& Williams, 2004; Wings, 2004). En la estructura mineral del apatito biológico las sustituciones isomórficas ocurren con el calcio, fosfato e hidroxilo conservando, generalmente, su morfología original. Si las aguas subterráneas están enriquecidas en carbonato, la mineralogía del hueso fósil pasará a ser un carbonato hidroxiapatito o dahllita $\left[\mathrm{Ca}_{5}\left(\mathrm{PO}_{4} \mathrm{CO}_{3}\right)_{3}(\mathrm{OH})\right]$ y si hay un aporte de flúor, éste se introducirá en la estructura mineral y dará lugar a un carbonato fluorapatito o francolita $\left[\mathrm{Ca}_{5}\left(\mathrm{PO}_{4} \mathrm{CO}_{3}\right)_{3}(\mathrm{~F})\right]$ (Hubert et al., 1996; Trueman, 1999; Merino, 2000).

Estos cambios composicionales, así como la precipitación de minerales en los poros de los huesos fósiles proporcionan información sobre la importancia de los procesos diagenéticos en la preservación del hueso y su relación con el ambiente deposicional (Huerta et al., 2001; Goodwin et al., 2007). Para Martin et al. (2005) la composición elemental de los huesos diagenéticamente alterados refleja la composición del agua del lugar de enterramiento.
Investigaciones científicas sobre el comportamiento de las tierras raras en restos de dinosaurios han revelado la importancia que tienen como indicadores de cambios en las condiciones paleoambientales (Pereda-Suberbiola et al., 2000; Samoilov et al., 2001; Lécuyer et al., 2003; Patrick et al., 2004). Diversos autores han realizado estudios sobre isótopos en el material óseo de dinosaurios (Barrick \& Showers, 1994; Goodwin et al., 2002; Showers \& Genna, 2002), así como de la preservación de los tejidos blandos (Schweitzer et al., 2007).

El registro paleontológico del Cretácico Inferior de la Serranía de Cuenca es conocido por tres importantes yacimientos: Las Hoyas, Uña y Buenache de la Sierra. El objetivo del presente trabajo es contribuir al conocimiento de la mineralogía y la composición elemental de restos fósiles de un ornitópodo de tipo iguanodóntido del yacimiento Buenache de la Sierra, con el fin de conocer mejor los procesos tafonómicos acaecidos y aportar nuevos datos para la reconstrucción paleoecológica de los yacimientos barremienses de Cuenca.

\section{Situación geológica}

El yacimiento Buenache de la Sierra está localizado en la Serranía de Cuenca, dentro de la llamada Cubeta de Buenache de la Sierra. El yacimiento pertenece a la Formación Calizas de la Huérguina que se extiende por toda el área central de la Cuenca Suroccidental Ibérica. La Formación Calizas de la Huérguina está compuesta por un gran número de facies depositadas en ambientes aluviales distales, palustres y lacustres durante el Barremiense superior (Vilas et al., 1982). El registro más potente de esta formación se encuentra en la Cubeta de Las Hoyas, cuyo registro sedimentario se puede dividir en cuatro secuencias deposicionales diferentes, separadas por discordancias o paraconformidades locales. De base a techo estas unidades han sido nombradas como: Rambla de las Cruces I, Rambla de las Cruces II, Pocillo del Pozuelo y Hoya de la Madre de Las Latas (Fregenal-Martínez, 1998; Fregenal-Martínez \& Meléndez, 2000). En la cubeta de Buenache de la Sierra solo se han reconocido las 


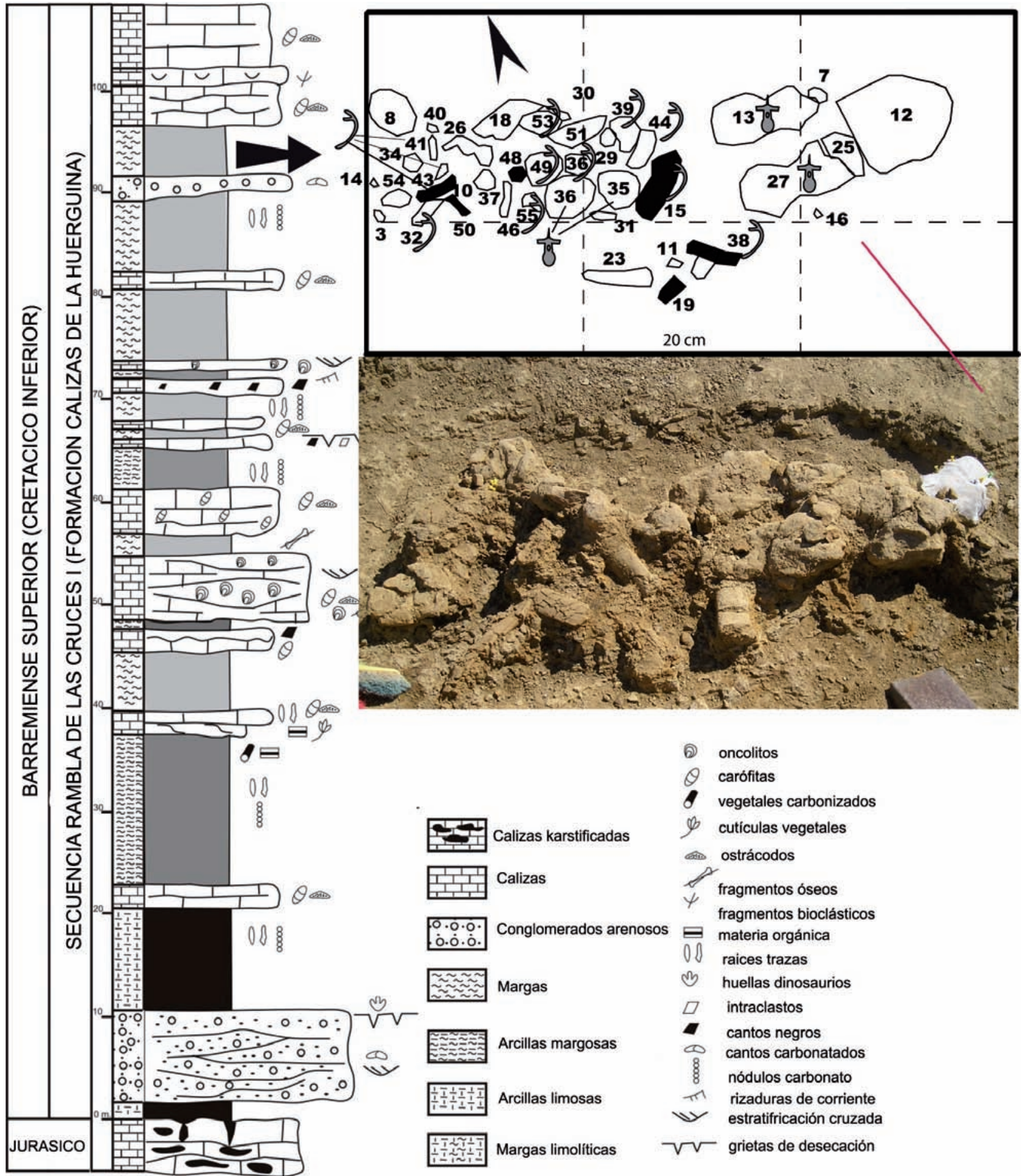

Fig. 1.-Columna de la Secuencia Rambla de las Cruces I en la cubeta de Buenache de la Sierra (según Buscalioni et al., 2008). La flecha señala la posición del yacimiento. Fotografía de la excavación del 2007 y mapa de cuadrícula con los restos numerados. En el mapa se han marcado en fondo negro los ejemplares analizados. En la parte inferior de la cuadrícula se ha representado las orientaciones de 19 restos con morfologías alargadas calculadas a partir del mapa de cuadrícula. El área de los segmentos es proporcional a la frecuencia. Cálculos realizados con el programa PAST (Hammer \& Harper, 2008). El p valor $(0,024)$ de la Chi $^{2}$ no permite rechazar la hipótesis nula de distribución al azar, aunque el número de elementos es escaso. 
dos secuencias inferiores. La secuencia Rambla de las Cruces I tiene una composición siliciclástica y carbonática caracterizada por una gran variedad de facies de llanura de inundación y canales que son equivalentes en número o incluso dominan sobre las facies carbonáticas palustres y lacustres (FregenalMartínez, 2007). Datos sedimentológicos paleogeográficos y apoyados en modelos generales paleoclimáticos indican que la sedimentación se produjo en un clima subtropical con alternacia de periodos secos y húmedos (Buscalioni et al., 2008). Los restos fósiles que se analizan se recogieron en un nivel de margas arenosas perteneciente a la secuencia de la Rambla de las Cruces I (Fig. 1). Este tipo de facies con abundantes restos de plantas y carbón vegetal son el resultado de la sedimentación en áreas de charcas estancadas en llanuras de inundación donde la acumulación de restos vegetales induce condiciones reductoras.

\section{Material y métodos}

El tercio inferior de la secuencia de La Rambla de las Cruces I, en la cubeta de Buenache de la Sierra, registra numerosas asociaciones de microfósiles (algunas muy ricas en microvertebrados) (Buscalioni et al., 2008). Hacia el techo de esta secuencia, es frecuente encontrar abundantes esquirlas de grandes huesos en superfice. Las excavaciones realizadas en 2007 dejaron al descubierto una asociación de grandes restos acumulados, formando una masa compuesta por vértebras aisladas y fragmentos de costillas tentativamente atribuidos a un dinosaurio ornitópodo (Fig. 2). El interés de este estudio radica en comprender si los fósiles de grandes dinosaurios muestran rasgos tafonómicos que permitieran caracterizarlos como alóctonos, pues las asociaciones de microfósiles estudiadas están compuestas por fósiles autóctonos o parautóctonos de taxones considerados como démicos (habitantes de charcas y lagunas). El material se encuentra en el Departamento de Paleontología de la Universidad Autónoma de Madrid, y será depositado en el Museo de las Ciencias de Castilla-La Mancha con la sigla BUEDIN2007.

Las vértebras encontradas son semejantes a las descritas por Francés \& Sanz (1989), que señalaron por primera vez la presencia de ornitópodos herbívoros de tipo "iguanodóntido" en esta localidad. La acumulación de grandes huesos se halló en un nivel de margas arenosas junto con restos vegetales (cutí-

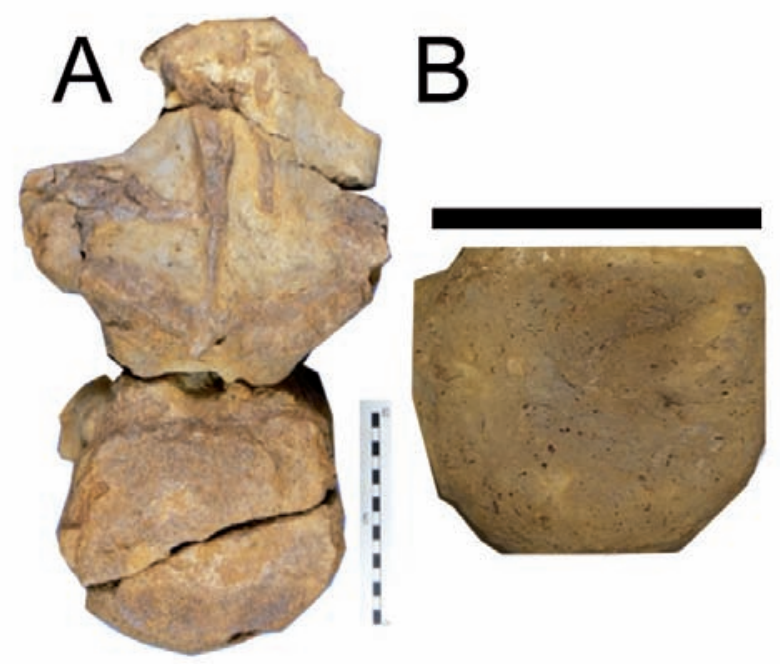

Fig. 2.-A. Vértebra N27 atribuida a Iguanodontia indet. Escala $15 \mathrm{~cm}$. B. centro vertebral N36 con restos de cutículas vegetales. Escala $8 \mathrm{~cm}$.

culas de coníferas tipo Frenelopsis, Fig. 2) y microfósiles (carófitas, ostrácodos, bivalvos, gasterópodos, fragmentos de cáscaras de huevos de arcosaurios y pequeños dientes de peces y cocodrilos).

Los macrorrestos no mostraban ninguna dirección predominante (Fig. 1) y estaban compuestos por 31 elementos óseos (13 fragmentos de costillas, 5 vértebras y fragmentos de huesos largos) y hasta 57 esquirlas de tamaño medio a pequeño $(10 \mathrm{~cm})$, distribuidas en unos $3 \mathrm{~m}^{2}$ de superficie. Los elementos estaban reagrupados siguiendo una distribución al azar, en un empaquetamiento donde los restos se hallaban en contacto entre sí en sucesivos microniveles. Algunos de los grandes huesos estaban envueltos en una costra ferruginosa compacta. El material fue preparado para su descripción tafonómica, exponiendo las alteraciones propias de la fase bioestratinómica (Fig. 3).

El material fósil analizado corresponde a cuatro fragmentos de costillas (N10, N15, N19 y N38) y a dos fragmentos de huesos indeterminados (N48 y N50). Se han realizado análisis en el sedimento encajante y en dos costillas de arcosaurios actuales: ave (Alopochen aegyptica) y caimán (Caiman crocodilus) procedentes de las colecciones de vertebrados fósiles y actuales de la Facultad de Ciencias Biológicas de la Universidad Autónoma de Madrid.

Las características microtexturales y mineralógicas de las muestras se han determinado mediante el estudio con microscopio petrográfico de seis lámi- 


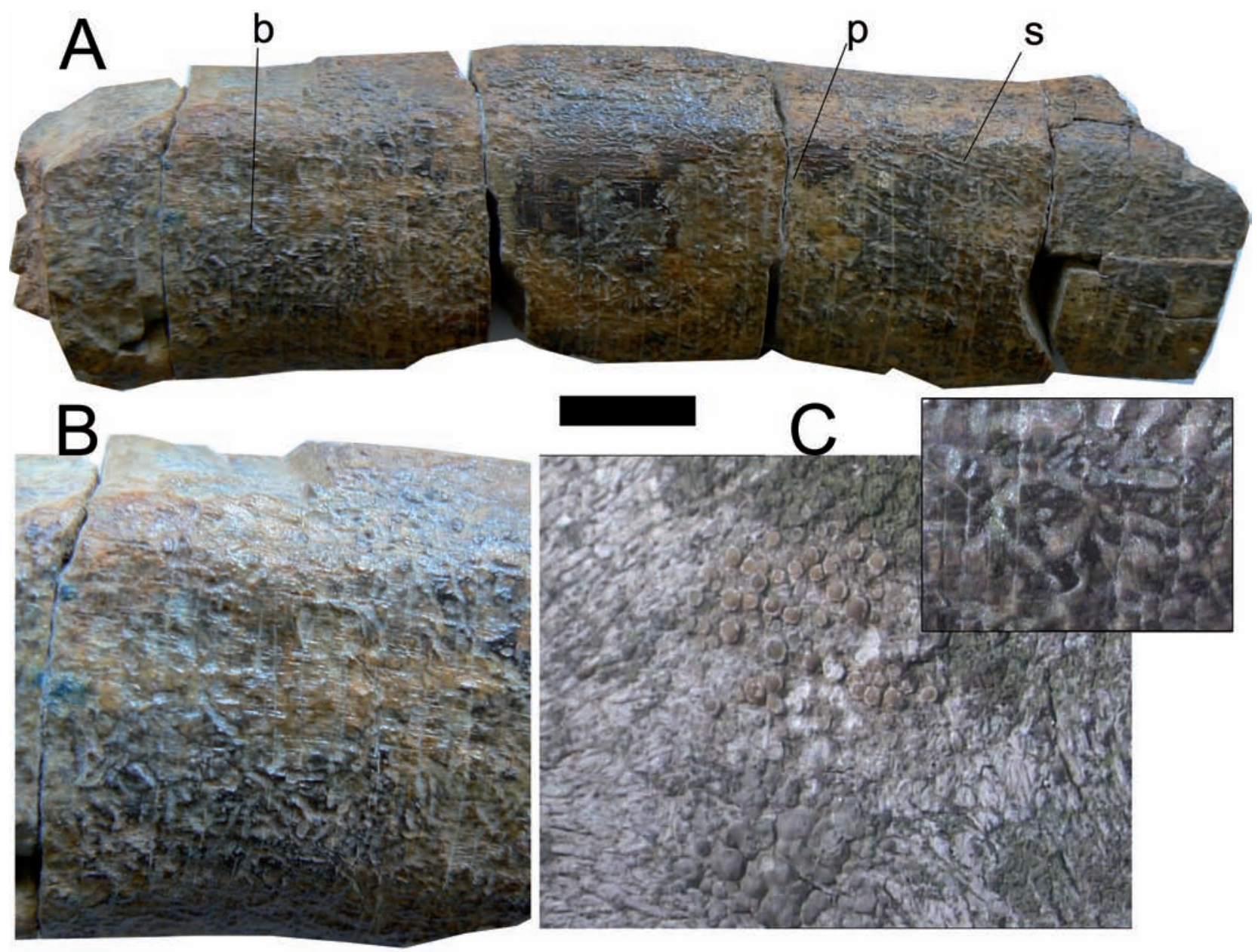

Fig. 3.-A. Resto N44 atribuido a una costilla, donde se han señalado las bioerosiones (b), la disposición en parche de las mismas (p) y las estrías de arrastre (s). Escala $2 \mathrm{~cm}$. B. Detalle de la ornamentación atribuida a bioerosión por líquenes. C. Detalle de la vértebra de un cánido actual procedente de Moralzarzal (Sierra de Madrid), donde se aprecian colonias de líquenes en la superficie. Nótese que se han retirado algunos de estas colonias para mostrar las oquedades resultantes en el hueso. En un cuadro en la parte superior detalle de estas señales en el hueso N44.

nas delgadas de $30 \mu \mathrm{m}$ de espesor. Para discriminar las fases minerales carbonáticas, las muestras se tiñeron selectivamente con alizarina roja y ferricianuro potásico, de acuerdo con el método de Lindhol y Finkelman (1972). También se ha utilizado el Microscopio Electrónico de Barrido modelo Quanta 200 con EDS (Energy-Dispersive X-ray Spectroscopy), ubicado en el Museo Nacional de Ciencias Naturales (MNCN) de Madrid.

Las distintas fases minerales de los componentes de las muestras y del sedimento se han identificado mediante técnicas de difracción de rayos X (DRX), en el rango comprendido entre $3-65^{\circ} 2 \theta$ a $2 \%$ min de velocidad. Para ello se ha utilizado un difractómetro Philips modelo PW 1710 equipado con monocro- mador de grafito y utilizando la radiación $\mathrm{Cu} \mathrm{K} \alpha$ también ubicado en el MNCN. Los registros de los difractogramas se han estudiado con el programa $X$ Powder (versión 2008). El índice de cristalinidad (IC) de las muestras actuales y fósiles se ha calculado según el método de Person et al., (1995), tomando los picos (202), (300), (211) y (112) localizados entre $30^{\circ}$ y $35^{\circ} 2 \theta$.

Se han realizado análisis químicos a 8 muestras de material óseo actual y fósil, así como a una muestra de sedimento. Los elementos mayoritarios y traza (21 en total) se obtuvieron con la microsonda electrónica Jeol JXA-8900 M del Centro de Microscopía Electrónica de la Universidad Complutense de Madrid. Las condiciones de 
N10

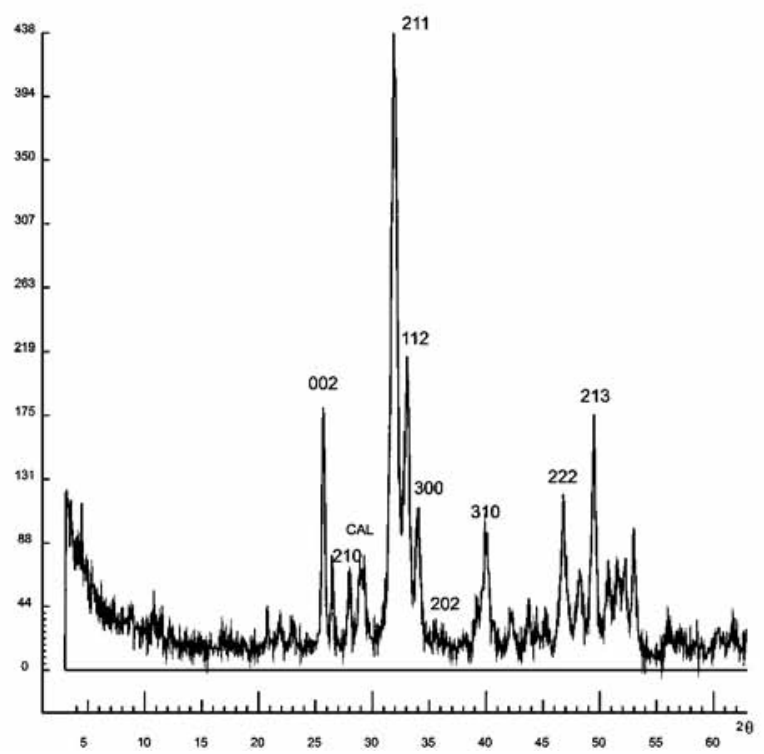

N15

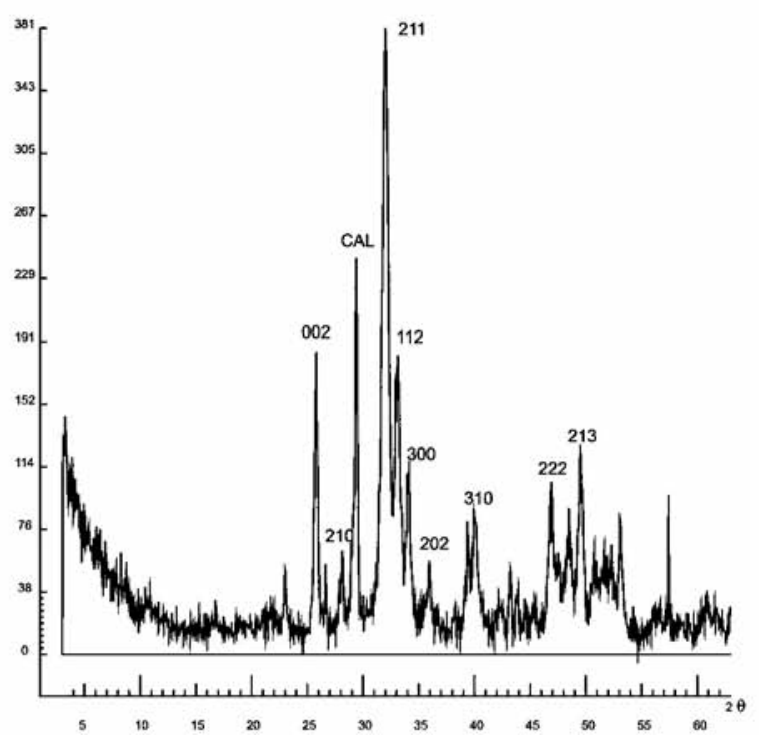

Fig. 4.-Difractogramas de RX de las muestras N10 y N15 correspondientes a costillas de dinosaurio ornitópodo del yacimiento Buenache de la Sierra. El fluorapatito es el componente mineral mayoritario. También aparecen picos de calcita.

funcionamiento fueron las siguientes: $15 \mathrm{Kv}$ de voltaje, $50 \mathrm{nA}$ de intensidad y diámetros de haz entre 1 y $5 \mu \mathrm{m}$.

Los análisis químicos y de difracción de rayos $\mathrm{X}$ de las muestras se realizaron únicamente sobre las partes de hueso compacto para evitar la presencia de impurezas en la medida de lo posible.

\section{Resultados}

\section{Alteraciones bioestratinómicas}

En general los huesos presentan bordes redondeados con abrasión y pulido de la superficie (Fig. 3). Los elementos están desarticulados y son fragmentarios, ni costillas ni huesos largos han conservado sus extremos distales y proximales. Aunque alguno de estos restos tiene fracturas oblicuas, las dominantes son transversales, perpendiculares al eje mayor del hueso, propias de la fase fosildiagenética, con grietas y fracturas netas, de bordes rectos. Las vértebras son los huesos fósiles más completos y conservan rasgos morfológicos que permiten su identificación taxonómica como Iguanodontia indet. (Fig. 2). Las vértebras están ligeramente comprimidas y han perdido sus extremos más conspicuos (espina neural y zigapófisis). Algunos centros muestran señales de meteorización, que aplicando los estadios propuestos por Behrensmeyer (1978) corresponderían al 2-3: superficie del hueso con escamas superficiales que en los bordes muestran una cierta profundidad dejando expuesta la estructura fibrosa del periostio. Las superficies de las costillas y de los fragmentos de huesos largos no están excesivamente meterorizadas y se encuentran en un estado 1 de meteorización con finas fisuras paralelas a las fibras de colágeno. En cambio, sus superficies están bioalteradas. En los fragmentos de mayor longitud (N24, hueso fósil indeterminado; N40, N44, N55 y N56 fragmentos de costillas) se aprecia en la capa más externa del periostio un patrón de oquedades anastomosadas que forman parches bien diferenciados. Estas oquedades resultan ser de un diámetro de 0,5-2 $\mathrm{mm}$ por lo que el agente biológico que las produjo podría asimilarse a parches de líquenes (Trudgill, 1988; Fernández-Jalvo et al., 2002; Hospitaleche et al., 2011). Este tipo de bioerosión se localiza preferentemente en una de las superficies. Algunas de estas marcas se proyectan en surcos someros similares a los producidos por musgos (Fig. 3). En todos los huesos se aprecian además series de finas estrías de arrastre. 

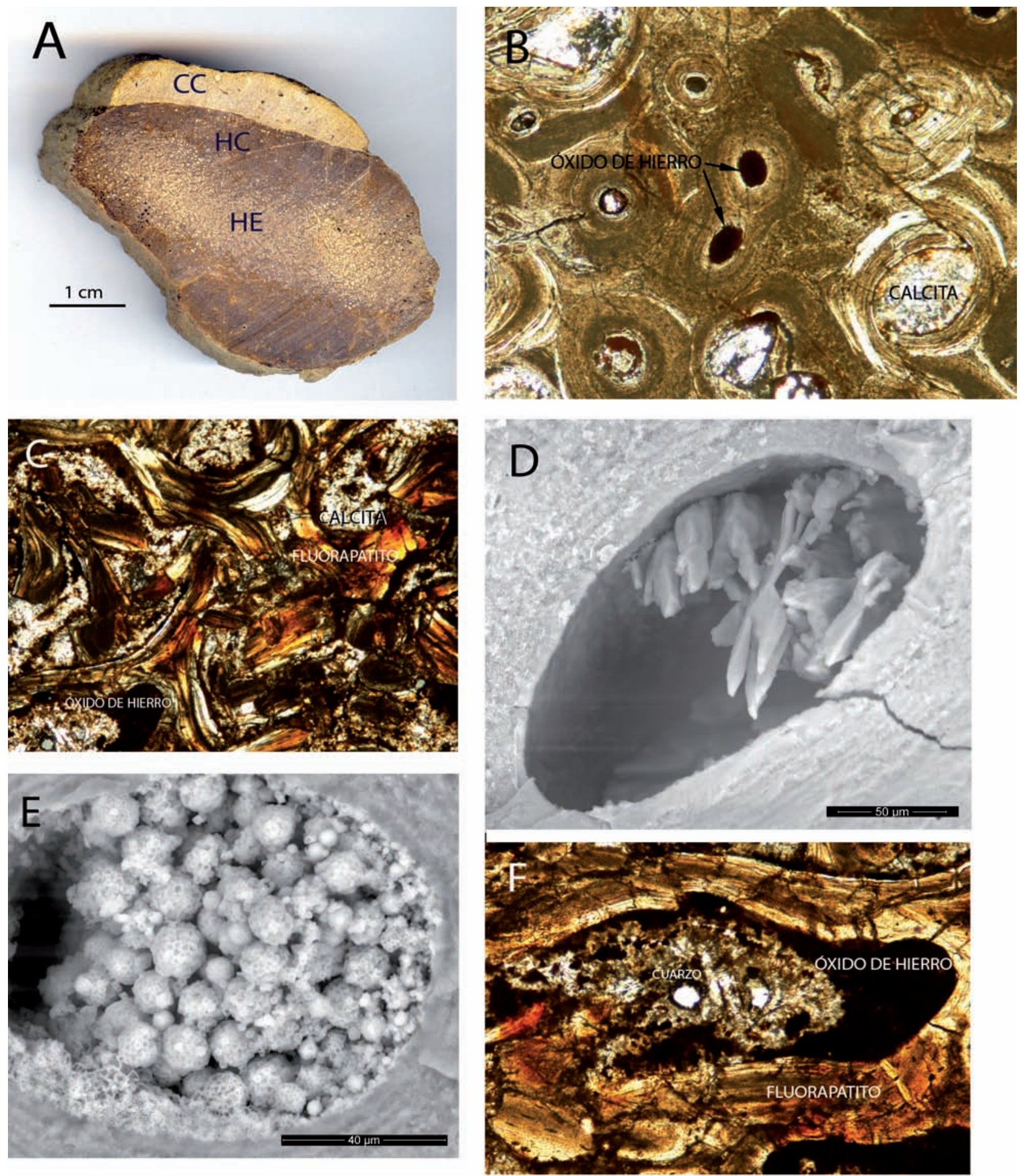

Fig. 5.-Muestra N10 correspondiente a una costilla de dinosaurio ornitópodo del yacimiento Buenache de la Sierra. A. Sección transversal en la que se observa una concreción carbonatada (CC), el hueso compacto (HC) y el esponjoso (HE). B. Microfotografía de canales de Havers rellenos de calcita y óxidos de hierro. Nícoles Paralelos (NP). C. Estructura esponjosa convertida en una brecha ósea. Nícoles Cruzados (NC). D. Imagen (MEB) de cristales de calcita rellenando parcialmente un canal de Havers. E. Framboides de óxidos de hierro en el interior de un poro. F. Estructura esponjosa rellena por un mosaico de calcita, cuarzo detrítico y óxidos de hierro. (NC). 


\section{Mineralogía e índice de cristalinidad (IC)}

Para el estudio de la mineralogía se han realizado análisis de difracción de rayos $\mathrm{X}$ a 6 muestras fósiles atribuidas a Iguanodontia (Fig. 4) y a 2 actuales, correspondientes a costillas de un caimán y de un ave. En las actuales, el hidroxiapatito o fosfato cálcico-hidróxido $\left[\mathrm{Ca}_{5}\left(\mathrm{PO}_{4}\right)_{3}(\mathrm{OH})\right]$ es la fase mineral predominante. En las muestras fósiles la mineralogía principal es el fluorapatito $\left[\mathrm{Ca}_{5}\left(\mathrm{PO}_{4}\right)_{3} \mathrm{~F}\right]$, lo que sugiere la presencia de procesos de sustitución postdeposicional del ión $\mathrm{OH}^{-}$original por el ión $\mathrm{F}^{-}$(Trueman, 1999; Merino \& Morales, 2008). El sedimento que engloba el material óseo, corresponde a margas arenosas.

El grado de perfección cristalográfica del apatito biológico se ha obtenido a través del índice de cristalinidad (IC) según Person et al., (1995). Las muestras actuales están pobremente cristalizadas $(0,14-0,21)$, mientras que las fósiles presentan un mayor grado de cristalinidad $(0,44-0,61)$. El grado de cristalinidad del apatito biológico parece aumentar con la incorporación del F- en la red cristalina (Schoeninger et al., 1989; Newesely, 1989) a la vez que parece disminuir con la incorporación del ión carbonato (Person et al., 1995), lo que confiere a los fósiles una mayor estabilidad y un decrecimiento de la solubilidad.

\section{Análisis petrográfico}

Los estudios petrográficos en láminas delgadas de las muestras son indispensables para la identificación de su mineralogía y textura y, por tanto, para la reconstrucción de la historia diagenética.

Un análisis petrográfico del material óseo fósil revela que hay dos grupos de muestras claramente diferenciados, tanto por sus características microanatómicas como por los diferentes procesos postsedimentarios experimentados. El primer grupo (muestras N10, N19, N38 y N50 - grupo A) se caracteriza por la presencia de framboides de óxidos de hierro y por el colapso de la estructura interna, mientras que el segundo grupo (muestras N15 y N48 - grupo B) presenta menor compactación mecánica y abundante cemento de calcita que sustituye parcialmente la estructura del hueso. A continuación se describen las muestras N10 y N15 como representativas de cada uno de los grupos.

\section{Descripción Grupo A (Muestra N10)}

En una sección transversal de la costilla se pueden observar dos zonas claramente diferenciadas en cuanto a sus texturas, una externa y otra interna (Fig. 5A). En algunas zonas el hueso esponjoso está en contacto con el sedimento encajante y falta parte del hueso compacto.

La pared externa o corteza es de color marrón oscuro, tiene unos $7 \mathrm{~mm}$ de espesor y corresponde al llamado hueso cortical o compacto. Las osteonas están bien preservadas, aunque algunas aparecen compactadas mecánicamente. Son de pequeño tamaño (entre $100 \mu \mathrm{m}$ y $400 \mu \mathrm{m}$ de diámetro) y se aprecian claramente sus estructuras de láminas o anillos concéntricos alrededor de los canales centrales. Algunos canales de Havers están rellenos total o parcialmente por un cemento micrítico de calcita y otros de óxidos de hierro y de una matriz siliciclástica (Fig. 5B). En algunos casos el óxido de hierro recubre las paredes internas de los poros. Se observan fracturas en el hueso compacto rellenas de óxido de hierro y cemento de calcita.

Hay una zona de transición del hueso compacto al esponjoso con estructura compacta, donde las cavidades van aumentando de tamaño hacia el interior del hueso. Estas cavidades están rellenas también, como en la zona anterior, de cemento calcítico y óxidos de hierro.

La zona interna presenta un moteado de color crema y corresponde al hueso trabecular o esponjoso de unos $10 \mathrm{~mm}$ de espesor. Aquí la estructura del hueso aparece colapsada, formando una brecha de esquirlas procedentes de los tabiques de la estructura ósea (Fig. 5C). Las cavidades están rellenas por un mosaico micrítico y microesparítico teñido por los óxidos de hierro que contiene como impurezas (Fig. 5D) y otras aparecen rellenas por arcilla. En el interior de algunos poros se observan framboides de óxidos de hierro (Fig. 5E), también aparecen granos de cuarzo dispersos (con un tamaño medio de 100 $\mu \mathrm{m})$ con las caras corroídas. Es común que el óxido de hierro (hematites) forme una franja alrededor de los poros y que aparezca también como islas debido al reemplazamiento que se ha llevado a cabo por cemento calcítico (Fig. 5F). La longitud de los poros está comprendida entre $2,7 \mathrm{~mm}$ y $120 \mu \mathrm{m}$.

\section{Interpretación Grupo A}

Las características diagenéticas muestran que contemporáneamente a la degradación de los teji- 

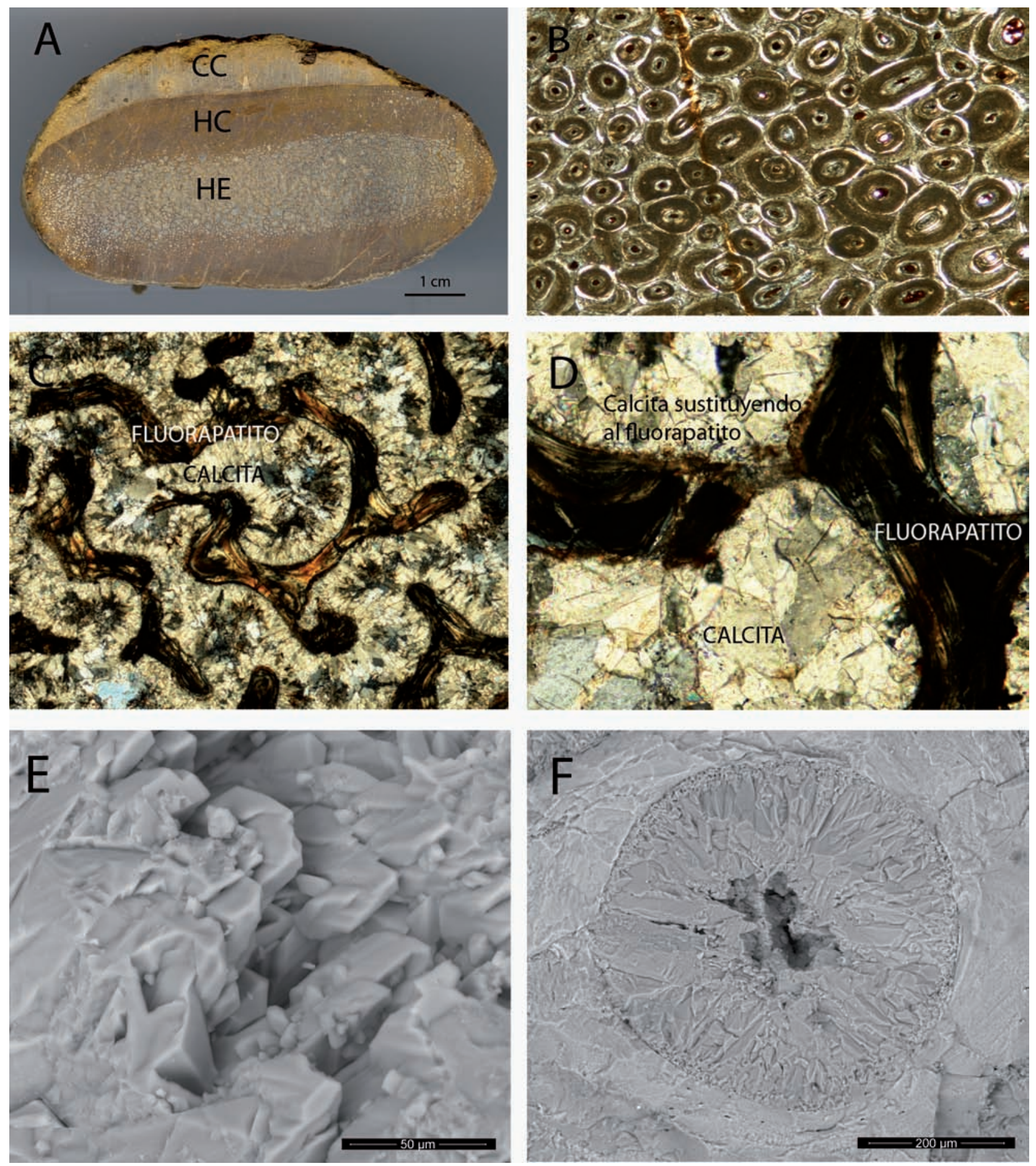

Fig. 6.-Muestra N15 correspondiente a una costilla de dinosaurio ornitópodo del yacimiento Buenache de la Sierra. A. Sección transversal en la que se observa una concreción carbonatada (CC), el hueso compacto (HC) y el esponjoso (HE). B. Microfotografía donde se observa la disposición de las osteonas en el hueso compacto. Nícoles cruzados (NC). C. Calcita rellenando los poros de la parte esponjosa del hueso fósil. Se aprecia una pérdida de la estructura histológica inicial por la precipitación de calcita. (NC). D. Detalle de la sustitución de fluorapatito por calcita. (NC). E. Microfotografía al MEB de una cavidad rellena por un mosaico esparítico. F. Detalle de un canal de Havers relleno parcialmente por cemento de calcita. 
dos blandos o inmediatamente después, la porosidad resultante se rellena parcialmente con partículas de cuarzo y arcillas en las primeras etapas de la fosilización, en o cerca de la superficie y en contacto aún con las aguas meteóricas.

Los framboides de óxidos de hierro que se observan en las cavidades del hueso proceden de piritas, e indican que en una etapa temprana de la diagénesis y en un ambiente reductor se formaron estos sulfuros de hierro (Coleman \& Raiswell, 1995) como consecuencia de la descomposición de la materia orgánica por la actividad bacteriana. Raiswell \& Canfield (1998) consideran que estos sulfuros se forman en sedimentos que están en un ambiente anóxico, por reacción del sulfato disuelto en el agua, que previamente se ha reducido por la actividad microbiana, con óxidos de hierro.

Posteriormente se pasó a una nueva etapa oxidante donde estos minerales autigénicos de hierro se oxidaron. Para que esta oxidación se llevara a cabo el nivel freático habría bajado, lo que se correspondería con un clima cálido y contrastado, sucediendo periodos húmedos a otros muy cálidos y secos, lo que concuerda con la interpretación dada por Buscalioni et al. (2008) para el yacimiento. La aparición de minerales ferrosos y posteriormente de óxidos de hierro formados a partir de los anteriores, es previo a la etapa de cementación calcítica. El cemento carbonático indica una fosilización poco profunda que correspondería a un medio subaéreovadoso, donde los poros están ocupados por aire y agua. Esta agua intersticial da lugar unos cementos de calcita cuya génesis se atribuye a múltiples centros de nucleación y a una cristalización muy rápida (Dunhan, 1971).

Las fracturas que se observan en la región cortical de los huesos fósiles, así como el colapsamiento de los tabiques del hueso esponjoso se deben a los esfuerzos de carga que ha sufrido el material óseo durante el enterramiento y a los procesos tectónicos sufridos. En estas fracturas han precipitado posteriormente minerales como óxidos de hierro y calcita.

\section{Descripción Grupo B (Muestra N15)}

El hueso compacto es de color marrón claro y tiene unos $7 \mathrm{~mm}$ de espesor (Fig. 6A). Los sistemas de Havers están bien preservados, aunque en algunos se aprecia una cierta deformación producida por la presión del enterramiento (Fig. 6B). Sus tamaños varían desde $100 \mu \mathrm{m}$ a $425 \mu \mathrm{m}$ de diámetro. El interior de los canales está relleno mayoritariamente por cemento calcítico (esparita), algunos granos detríticos de cuarzo y óxidos de hierro en pequeña proporción. Las fracturas que recorren el hueso compacto están rellenas de cristales de calcita.

Por otra parte, el hueso esponjoso presenta un moteado de color gris y tiene unos $12 \mathrm{~mm}$ de espesor. Conserva relativamente bien su estructura, aunque la calcita sustituye parcialmente al fluorapatito (Fig. 6C). Los poros están totalmente ocluidos por un cemento de calcita micrítica que rodea las paredes y sobre éste, aparecen cristales de esparita limpios, que van aumentando de tamaño hacia el centro de los poros (entre $500 \mu \mathrm{m}$ y $800 \mu \mathrm{m}$ de diámetro), produciendo un mosaico tipo blocky (Fig. 6D). Los granos de cuarzo dispersos y los óxidos de hierro se introdujeron en los huesos a través de los poros y fracturas durante el enterramiento, favorecidos por las aguas de infiltración. No se observa ningún colapsamiento de los tabiques como en la muestra N10.

\section{Interpretación Grupo B}

Los signos diagenéticos muestran que el material óseo inicialmente fue enterrado a poca profundidad, lo que correspondería con un medio vadoso en el que se formó un cemento micrítico discontinuo. Seguidamente estos huesos sufrieron un nuevo proceso diagenético al pasar a una zona saturada activa, donde los poros se rellenaron de una solución con una alta concentración de carbonato cálcico. Sobre el cemento vadoso empiezan a nuclear unos cristales de esparita (Fig. 6E) que terminan sellando completamente las cavidades. Loucks (1977) señaló que el agrandamiento de los cristales hacia el centro de los poros es una característica distintiva de cementación freática, con enterramiento poco profundo en un ambiente continental. Posteriormente, en la última etapa los huesos pasaron a una zona subaérea, como se aprecia por la aparición de algunos poros debido a la disolución del cemento en el centro de los canales de Havers (Fig. 6F).

\section{Composición geoquímica}

Se han realizado análisis químicos con la microsonda electrónica a 4 costillas y a 2 fragmentos óseos indeterminados de dinosaurio y a 2 costillas actuales de ave y de caimán (Tabla 1). El objeto de 
Tabla 1.-Resultados de los análisis químicos del sedimento y de las muestras actuales y fósiles del yacimiento Buenache de la Sierra (Barremiense superior, Formación Calizas de la Huérguina).

\begin{tabular}{|c|c|c|c|c|c|c|c|c|c|}
\hline & CAI & ALO & N10 & N15 & N19 & N38 & N48 & N50 & SED \\
\hline $\mathbf{A l}_{2} \mathbf{O}_{3}$ & 0,421 & 1,223 & 0,213 & 0,049 & 0,091 & 0,039 & 0,080 & 0,019 & 3,096 \\
\hline MnO & 0,008 & 0,000 & 0,000 & 0,064 & 0,040 & 0,012 & 0,000 & 0,068 & 0,057 \\
\hline $\mathrm{CaO}$ & 38,527 & 44,890 & 48,984 & 51,111 & 49,688 & 50,648 & 47,500 & 49,275 & 44,989 \\
\hline $\mathbf{P}_{2} \mathbf{O}_{5}$ & 28,351 & 31,485 & 33,957 & 35,587 & 34,800 & 34,569 & 32,497 & 33,012 & 0,155 \\
\hline $\mathrm{Na}_{2} \mathrm{O}$ & 0,635 & 0,816 & 0,526 & 0,632 & 0,552 & 0,554 & 0,448 & 0,510 & 0,008 \\
\hline $\mathrm{SiO}_{2}$ & 0,000 & 0,000 & 0,000 & 0,000 & 0,000 & 0,000 & 0,000 & 0,000 & 4,863 \\
\hline $\mathrm{FeO}$ & 0,011 & 0,018 & 0,195 & 0,262 & 1,210 & 1,692 & 0,118 & 1,024 & 2,520 \\
\hline $\mathbf{K}_{2} \mathbf{O}$ & 0,110 & 0,000 & 0,000 & 0,000 & 0,016 & 0,013 & 0,000 & 0,000 & 0,245 \\
\hline $\mathrm{SO}_{3}$ & 0,349 & 0,354 & 0,693 & 0,952 & 0,900 & 1,213 & 0,875 & 1,037 & 0,033 \\
\hline MgO & 0,546 & 0,432 & 0,050 & 0,126 & 0,085 & 0,102 & 0,078 & 0,068 & 0,234 \\
\hline SrO & 0,193 & 0,072 & 0,138 & 0,273 & 0,278 & 0,290 & 0,229 & 0,248 & 0,000 \\
\hline $\mathrm{CuO}$ & 0,000 & 0,000 & 0,000 & 0,035 & 0,000 & 0,000 & 0,003 & 0,000 & 0,000 \\
\hline $\mathrm{TiO}_{2}$ & 0,023 & 0,016 & 0,033 & 0,010 & 0,022 & 0,022 & 0,000 & 0,031 & 0,114 \\
\hline Cl & 0,038 & 0,050 & 0,017 & 0,010 & 0,022 & 0,019 & 0,013 & 0,041 & 0,033 \\
\hline $\mathbf{F}$ & 1,257 & 0,459 & 3,254 & 2,994 & 2,991 & 3,092 & 2,826 & 2,796 & 0,033 \\
\hline $\mathrm{ZnO}$ & 0,029 & 0,158 & 0,155 & 0,000 & 0,095 & 0,000 & 0,044 & 0,073 & 0,075 \\
\hline $\mathrm{Cr}_{2} \mathrm{O}_{3}$ & 0,057 & 0,061 & 0,019 & 0,009 & 0,009 & 0,000 & 0,070 & 0,025 & 0,031 \\
\hline $\mathrm{BaO}$ & 0,083 & 0,046 & 0,002 & 0,000 & 0,000 & 0,026 & 0,000 & 0,000 & 0,028 \\
\hline $\mathrm{NiO}$ & 0,016 & 0,060 & 0,000 & 0,008 & 0,003 & 0,041 & 0,055 & 0,005 & 0,000 \\
\hline $\mathbf{V}_{2} \mathbf{O}_{3}$ & 0,034 & 0,024 & 0,000 & 0,049 & 0,008 & 0,000 & 0,000 & 0,011 & 0,002 \\
\hline $\mathrm{CoO}$ & 0,021 & 0,000 & 0,000 & 0,000 & 0,128 & 0,000 & 0,000 & 0,013 & 0,022 \\
\hline
\end{tabular}

Los elementos mayores y trazas vienen dados en porcentajes (\%). Abreviaturas: CAI, Costilla de Caimán; ALO, Costilla de ave; $\mathrm{N} 10, \mathrm{~N} 15, \mathrm{~N} 19$ y N38, fragmentos de costillas de dinosaurio; N48 y N50, fragmentos de huesos indeterminados de dinosaurio; SED, sedimento.

estos análisis ha sido el de comparar la ganancia o pérdida de elementos que ha tenido lugar en el material óseo fósil respecto al actual, y de esa manera conocer mejor los procesos fosildiagenéticos. También se ha analizado el sedimento adyacente para determinar cómo influye en la composición de las muestras.

Los huesos tienen una estructura porosa y, por tanto, son susceptibles de la entrada o salida de iones, así como de la precipitación de minerales en las cavidades (Trueman et al., 2006).

Se puede observar en la figura 7 que el calcio y el fósforo son los componentes mayoritarios de los huesos, tanto actuales como fósiles, produciéndose un enriquecimiento diagenético en los dos. El calcio es un elemento que es sustituido fácilmente por otros en la estructura del apatito biológico, pero parte de su contenido procede de la precipitación de calcita en los poros (Sillen, 1981). Es el elemento mayoritario en el sedimento que envuelve el material óseo $(44,99 \%)$. También, otros elementos mayoritarios como el hierro, azufre, flúor manganeso y estroncio experimentan, en general, incrementos composicionales en el material óseo fósil. El contenido de hierro en las muestras puede ser debido a la precipitación de óxidos de hierro procedentes del sedimento circundante y a la oxidación de las piritas formadas anteriormente en un ambiente reductor (Fig. 5E).

Por el contrario, se aprecia una pérdida de aluminio (aunque es abundante en el sedimento), cloro, magnesio y sodio en el material óseo fósil respecto al actual. El potasio también disminuye durante la fosildiagénesis, si se compara con la muestra actual CAI. La sílice forma parte de la composición del sedimento adyacente $(4,86 \%)$ pero no del hueso cortical analizado. Aparece en los poros del hueso fósil esponjoso, en forma de granos detríticos de cuarzo, procedentes del sedimento y depositados mecánicamente dentro de él. No se aprecia cuarzo autigénico en ninguno de los huesos examinados. 


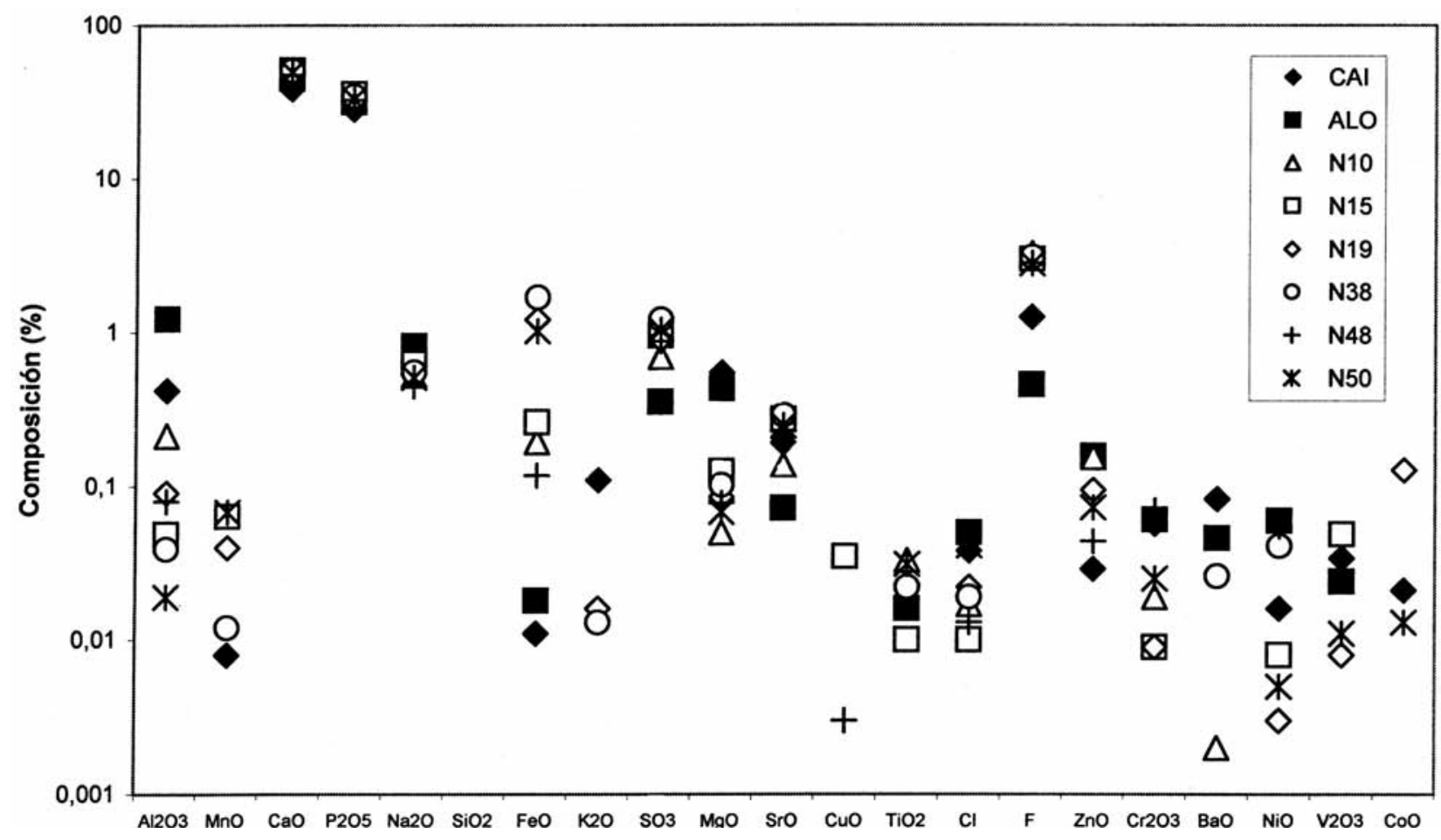

Fig. 7.-Distribución de los resultados analíticos de los componentes elementales, correspondientes a las muestras actuales (CAI y ALO) y fósiles de dinosaurio (N10, N15, N19, N38, N48 y N50) del yacimiento de Buenache de la Sierra (Cuenca).

En lo que respecta a los elementos traza se observa, en general, una pérdida diagenética de bario, cromo y vanadio (Fig. 7). El resto de los elementos muestran comportamientos diferentes en cuanto a su composición, dependiendo de las muestras.

Las variaciones composicionales y mineralógicas que han sufrido los huesos a lo largo de su historia diagenética son complejas. Para determinar el comportamiento de las muestras a partir de su composición química, se ha aplicado un tratamiento estadístico multivariante (análisis de componentes principales) utilizando el programa SPSS v.17.0 (2008).

Como se observa en la figura 8 las muestras fósiles se han diferenciado en dos grupos, el A que corresponde a las muestras N10, N38, N50 y N19, y el B a las muestras N15 y N48. Las actuales aparecen significativamente alejadas de las fósiles.

Se puede interpretar las distancias del material óseo en el gráfico en términos de diferencias composicionales, atribuidas, en el caso de los fósiles, a los distintos procesos fosildiagenéticos que han sufrido. Estos dos grupos se corresponden con los grupos A y B definidos mediante el estudio petrográfico.

\section{Interpretación tafonómica}

Los grandes huesos de Iguanodontia se produjeron en ambientes subaéreos, con una exposición relativamente corta, que indujo a una suave meteorización y bioerosión por raíces y líquenes. La diferente composición química de las muestras junto con el desgaste del hueso, con contornos redondeados, abrasión y marcas de arrastre, así como la falta de conexión anatómica de las piezas fracturadas parece indicar que los restos sufrieron transporte. Por tanto, los fósiles pertenecerían a individuos diferentes y después de producidos fueron removilizados. Analizando la relación de los cementos carbonáticos con los demás procesos diagenéticos acaecidos en los dos grupos A y B, se observa que han tenido historias tafonómicas distintas previas al enterramiento final. En ambos grupos tanto los procesos diagenéticos tempranos como los posteriores son distintos. Es de destacar que las muestras del grupo A presentan un colapso notable en su estructura interna que no se aprecia en los del grupo B. Estos restos resedimentados producirían posteriormente la asociación mezclada que encontramos en el yacimiento, formada por elementos con entidades 
biológicas de ambientes diferentes (terrestres: plantas y dinosaurios; semiacuáticos: cocodrilos; acuáticos: peces, carófitas, ostrácodos, gasterópodos y bivalvos). Los elementos resedimentados fueron reagrupados y empaquetados (siguiendo una distribución al azar) en una charca o laguna somera y expuestos a cambios del nivel freático.

\section{Conclusiones}

Al comparar las muestras fósiles de dinosaurio del yacimiento Buenache de la Sierra con las actuales, se ha observado que en el material óseo fósil se han producido importantes cambios mineralógicos y geoquímicos a lo largo de su historia diagenética.

En las costillas de los taxones actuales estudiados, la fase mineral predominante es el hidroxiapatito y en las fósiles el fluorapatito. Este cambio mineralógico que se ha producido en las muestras fósiles, se debe al intercambio de elementos en la estructura del apatito biológico. El mineral autigénico más abundante en las muestras es la calcita, que aparece rellenando las cavidades óseas y fracturas.

El calcio y el fósforo son los elementos mayoritarios en los huesos actuales y fósiles. En los dos se observa un enriquecimiento en las muestras fósiles. El hierro, azufre, flúor y estroncio también registran una ganancia, sin embargo, para el aluminio, cloro, magnesio y sodio se aprecia una pérdida. Algunos elementos traza como el bario, cromo y vanadio, en general, sufren una pérdida diagenética y el resto se comportan de diferentes formas según las muestras. La composición elemental del sedimento adyacente no determina en todos los casos un enriquecimiento o pérdida de los elementos químicos en las muestras.

Al principio del enterramiento, en las muestras N10, N19, N38 y N50 (Grupo A) se introducen mecánicamente por los poros y fracturas granos de cuarzo y arcilla por el efecto de las aguas de infiltración, así como óxidos de hierro procedentes de la alteración de minerales constituyentes de la roca encajante. Seguidamente, y en una etapa temprana, pasaron a unas condiciones reductoras, donde el $\mathrm{Fe}^{2+}$ dio lugar a la precipitación de piritas. En una etapa posterior y debido a la modificación del nivel freático, cambian las condiciones reductoras a oxidantes y, por tanto, se alteran los minerales ferrosos y comienza la precipitación de calcita. La estructura del hueso esponjoso está colapsada formando una brecha de esquirlas.

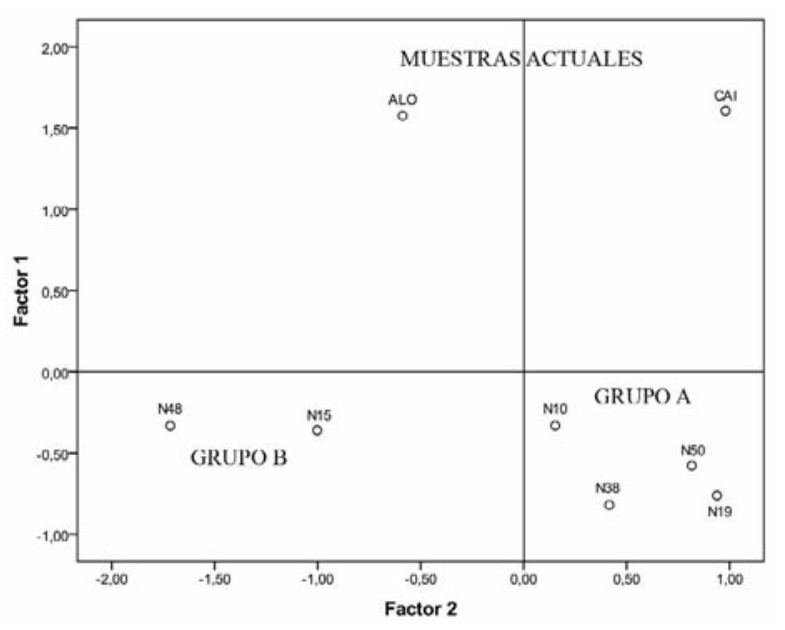

Fig. 8.-Representación de las muestras estudiadas, agrupadas en función de los dos factores discriminantes resultantes del análisis de los componentes principales. Se pueden diferenciar dos grupos de muestras fósiles (A y B).

En los poros de los huesos fósiles correspondientes a las muestras N15 y N48 (Grupo B) aparecen óxidos de hierro (en pequeña proporción) y granos de cuarzo dispersos, que fueron introducidos gravitacionalmente por el agua de infiltración durante el enterramiento. Seguidamente, en una etapa diagenética temprana y en un medio vadoso, tuvo lugar la precipitación de un cemento micrítico en los poros. Cuando estas condiciones cambian y se pasa a una zona freática se produce la precipitación de un mosaico de esparita oclusivo. La estructura esponjosa se conserva aunque la calcita sustituye parcialmente al fluorapatito que forma los tabiques.

Los huesos fósiles analizados tuvieron historias tafonómicas distintas. Estos elementos fueron transportados y resedimentados depositándose en las charcas de las llanuras de inundación que constituían los ambientes propios del humedal de La Huérguina.

\section{AGRADECIMIENTOS}

Nuestro agradecimiento a Rafael González, del Laboratorio de Difracción de Rayos X del MNCN (CSIC), Manuel Castillejo del MNCN por la preparación de las láminas delgadas y a Alberto Jorge del Laboratorio de Microscopía del MNCN. A Jorge Morales, Manuel Salesa y a Javier García Guinea por su colaboración, y Alfredo Fernández del Centro de Microscopía Electrónica de la UCM. Agradecemos a Belén Muñoz del Departamento de Estratigrafía de la UCM sus comentarios, así como a Xavier Pereda de la Universidad del País Vasco. Fernando del Museo de los Zoolitos de Buenache colaboró en el 
hallazgo del material. Este trabajo se ha realizado en el marco de los proyectos: CCGL2009-11838-BTE, MICINN y CGL2008-05813-CO2-01, MICINN (Spanish Government) and The Research Group CAM-UCM 910607.

\section{Referencias}

Aguinaga, I., Manso, E., Guillén, F., Viñes, J.J., Guillén, J.J., Martínez, M.J., Pérez de Ciriza, J.A., Brun, C., Marín, B., Herrera, T., García-Marco, L., Bayo, J. \& grupo EPLODIN, (2000). Estudio de la acumulación de plomo en dientes primarios. Anales del Sistema Sanitario de Navarra, 23 (1).

Barrick, R.E. \& Showers, W.J. (1994). Thermophysiology of Tyrannosaurus rex: evidence from oxygen isotopes. Science, 265: 222-224. doi:10.1126/science.265.5169.222

Behrensmeyer, A.K. (1978). Taphonomic and ecologic information from bone weathering. Paleobiology, 4: 150-162.

Buscalioni, A.D., Fregenal-Martínez, M.A., Bravo, A., Poyato-Ariza, F.J., Sanchíz, B., Báez, A.M., Cambra Moo, O., Martín Closas, C., Evans, S.E. \& Marugán Lobón, J. (2008). The vertebrate assemblage of Buenache de la Sierra (Upper Barremian of Serranía de Cuenca, Spain) with insights into its taphonomy and palaeoecology. Cretaceous Research, 29: 687-710. doi:10.1016/j.cretres.2008.02.004

Coleman, M.L. \& Raiswell, R. (1995). Source of carbonate and origin of zonation in pyritiferous carbonate concretions: evaluation of a dynamic model. American Journal of Science, 298: 282-308. doi:10.2475/ ajs.295.3.282

Dauphin, Y. \& William, C.T. (2004). Diagenetic trends of dental tissues. Comptes Rendus Palevol, 3: 583-590. doi:10.1016/j.crpv.2004.07.007

Driessens, F.C.M. \& Verbeeck, R.M.H. (1990). Biominerals. CRC Press, Boca Raton, Florida.

Fernández-Jalvo, Y., Sanchez-Chillón, B., Andrews, P., Fernández-López, S. \& Alcalá Martínez, L. (2002). Morphological taphonomic transformations of fossil bones in contiental environments and repercussions on their chemical composition. Archaeometry, 44: 353361. doi:10.1111/1475-4754.t01-1-00068

Francés, V. \& Sanz, J. L. (1989). Restos de dinosaurios del Cretácico Inferior de Buenache. In: La Fauna del Pasado en Cuenca: I Curso de Paleontología (Sanz, J. L., coord.). Instituto Juan de Valdés, Excmo Ayuntamiento de Cuenca, 125-144.

Fregenal-Martínez, M.A. (1998). Análisis de la cubeta sedimentaria de Las Hoyas y su entorno paleogeográfico (Cretácico Inferior, Serranía de Cuenca). Sedimentología y aspectos tafonómicos del yacimiento de Las Hoyas. Tesis Doctoral, Universidad Complutense de Madrid, 354 pp.

Fregenal-Martínez, M.A. (2007). Lagos, humedales y ecosistemas del Barremiense (Cretácico Inferior) de la Serranía de Cuenca . Visita a los yacimientos de Bue- nache de la Sierra y Las Hoyas. En Cantera Paleontógica (Cambra-Moo, O., Martinez Pérez, C., Chamero, B., Escaso, F., Esteban, S. \& Marugán-Lobón, J. , eds.). Ediciones Provinciales, 53: 381-396.

Fregenal-Martínez, M.A. \& Meléndez, N. (2000). The lacustrine fossiliferous deposits of the Las Hoyas subbasin (Lower Cretaceous, Serranía de Cuenca, Iberian Ranges, Spain). In:. Lake basins through space and time (Gierlowski-Kordesch, E.H., Kelts, K., eds.). AAPG Studies on Geology, 46: 303-314.

Goodwin, M.B., Bench, G. \& Grant, P.G. (2002). Comments on "Isotopic analysis of dinosaur bones". Analytical Chemistry, 74 (13): 351A. doi:10.1021/ ac022059p

Goodwin, M.B., Grant, P.G. Bench, G. \& Holroyd, P.A. (2007). Elemental composition and diagenetic alteration of dinosaur bone: Distinguishing micron-scale spatial and compositional heterogeneity using PIXE. Palaeogeography, Palaeoclimatology, Palaeoecology, 253: 458-476. doi:10.1016/j.palaeo.2007.06.017

Hammer, $\varnothing$ \& Harper, D.A.T. (2008). Paleontological data analysis. Blackwell Publishing, 368 pp.

Henderson, P., Marlow, C.A., Molleson, T.I., Williams, C.T. (1983). Patterns of chemical change during fossilization. Nature, 306: 358-360. doi:10.1038/306358a0

Hospitaleche, C.A., Márquez, G., Martín-Pérez, L., Rosado, V. \& Cione, A.L. (2011). Lichen bioerosion on fossil vertebrates from the Cenozoic of Patagonia and Antarctica. Ichnos, 18: 1-8. doi:10.1080/10420940.2011.552577

Hubert, J.F., Panish, P.T., Chure, D.J. \& Prostak, K.S. (1996). Chemistry, microstructure, petrology, and diagenetic model of Jurassic dinosaur bones, Dinosaur National Monument, Utah. Journal of Sedimentary Research, 66: 531-547.

Huerta, P., Armenteros, I., Tesón, E. \& Khawam, P. (2001). Procesos diagenéticos que condicionan la preservación de huesos de dinosaurio (Cuenca de Cameros Sector Occidental). II Jornadas de Paleontología de Dinosaurios y su Entorno. Salas de los Infantes (Burgos, España), 171-182.

Lécuyer, C., Bogey, C., García, J.P., Grandjean, P., Barrat, J.A., Bardet, N. \& Pereda-Superbiola, X. (2003). The stable isotope composition and rare earth element content of vertebrate remains from the late Cretaceous of northern Spain (Laño): did the environmental record survive? Palaeogeography, Palaeoclimatology, Palaeoecology, 193: 457-471. doi:10.1016/S0031-0182(03)00261-X

Loucks, R.G. (1977). Porosity development and distribution in shoal-water carbonate complexes-subsurface Pearsall Formation (Lower Cretaceous) south Texas. Econ. Geology, Report of Investigations, 89: 97-126.

Martin, J.E., Patrick, D., Kihm, A.J., Foit Jr., F.F. \& Grandstaff, D.E. (2005). Lithostratigraphy, tephrochronology, and rare earth element geochemistry of fossils at the classical Pleistocene Fossil Lake area, south central Oregon. Journal of Geology, 113: 139-155. doi: $10.1086 / 427665$ 
Merino, L. (2000). Mineralogía y geoquímica del esqueleto de mamíferos del Neógeno español. Consejo Superior de Investigaciones Científicas, Madrid, $245 \mathrm{pp}$.

Merino, L. \& Morales, J. (2008). Relación del índice de cristalinidad (IC) con la edad y el contenido de iones F y $\mathrm{CO} 3$ en muestras de vertebrados fósiles. Estudios Geológicos, 64: 75-87. doi:10.3989/egeol.08641364

Moore, M.R., Campbell, B.C., Meredith, P.A., Beattie, A.D., Goldberg, A. \& Campbell, D. (1978). The association between lead concentrations in teeth and domestic water lead concentrations. Clinica Chimica Acta, 87: 77-83. doi:10.1016/0009-8981(78)90060-8

Newesely, H. (1989). Fossil bone apatite. Applied Geochemistry, 4: 233-245. doi:10.1016/08832927(89)90023-1

Patrick, D., Martin, J.E., Parris, D.C. \& Grandstaff, D.E. (2004). Paleoenvironmental interpretations of rare earth element signatures in mosasaurs (reptilia) from the upper Cretaceous Pierre Shale, central South Dakota, USA. Palaeogeography, Palaeoclimatology, Palaeoecology, 212: 277-294.

Pereda-Suberbiola X., Astibia H., Murelaga X., Elorza J.J. \& Gómez-Alday J.J. (2000). Taphonomy of the Late Cretaceous dinosaur-bearing beds of the Laño Quarry (Iberian Peninsula). Palaeogeography, Palaeoclimatology, Palaeoecology, 157 (3-4): 247-275. doi:10.1016/S0031-0182(99)00169-8

Person, A., Bocherens, H., Saliège, J. F., Paris, F., Zeitoun, V. \& Gérard, M. (1995). Early diagenetic evolution of bone phosphate: an X-ray diffractometry analysis. Journal of Archaeological Science, 22: 211-221. doi: $10.1006 /$ jasc. 1995.0023

Raiswell, R. \& Canfield, D.E. (1998). Sources of iron for pyrite formation in marine sediments. American Journal of Science, 298: 219-245. doi:10.2475/ ajs.298.3.219

Samoilov, V.S., Benjamini, Ch. \& Smirnova, E.V. (2001). Early diagenetic stabilization of trace elements in reptile bone remains as an indicator of Maastrichtian-Late Paleocene climatic changes: evidence from the Naran Bulak locality, the Gobi Desert (South Mongolia). Sedimentary Geology, 143: 15-39. doi:10.1016/S0037-0738(00)00192-5
Schoeninger, M.J.; Moore, K.M.; Murray, M.L. \& Kingston, J.D. (1989). Detection of bone preservation in archaeological and fossil samples. Applied Geochemistry, 4: 281-292. doi:10.1016/08832927(89)90030-9

Schweitzer, M.H., Wittmeyer, J.L. \& Horner, J.R. (2007). Soft tissue and cellular preservation in vertebrate skeletal elements from the Cretaceous to the present. Proceedings of the Royal Society. Biological Sciences, 274: 183-197. doi:10.1098/rspb.2006.3705

Showers, W.J. \& Genna, B. (2002). Reply to comments of "Isotope Analysis of Dinosaur Bones". Analytical Chemistry, 352A

Sillen, A. (1981). Post-depositional changes in Natufian and Aurignacian faunal bones from Hayonim Cave. Paleorient, 7: 81-85. doi:10.3406/paleo.1981.4300

SPSS (2008). SPSS para Windows. Versión 17.0: Chicago, Illinois.

Trueman, C.N. (1999). Rare earth element geochemistry and taphonomy of terrestrial vertebrate assemblages. Palaios, 14: 555-568. doi:10.2307/3515313

Trueman, C.N., Behrensmeyer, A.K., Potts, R. \& Tuross, N. (2006). High-resolution records of location and stratigraphic provenance from the rare earth element composition of fossil bones. Geochimica et Cosmochimica Acta, 70: 4343-4355.

Trudgill, S. (1988). Integrated geomorphological and ecological studies on rocky shores in southern Britain. Field Studies, 7: 239-279. doi:10.1016/j.gca.2006.06.1556

Vilas, L., Mas, R., García, A., Arias, C., Alonso, A., Meléndez, N., Rincon, R. con la colaboración de Elizaga, E., Fernández Calvo, C., Gutiérrez, C., y Meléndez, F. (1982). In: El Cretácico de España. Editorial de la Universidad Complutense de Madrid, 457-513.

Wings, O. (2004). Authigenic minerals in fossil bones from the Mesozoic of England: poor correlation with depositional environments. Palaeogeography, Palaeoclimatology, Palaeoecology, 204: 15-32. doi:10.1016/S0031-0182(03)00709-0

Recibido el 18 de octubre de 2012 Aceptado el 30 de mayo de 2013 